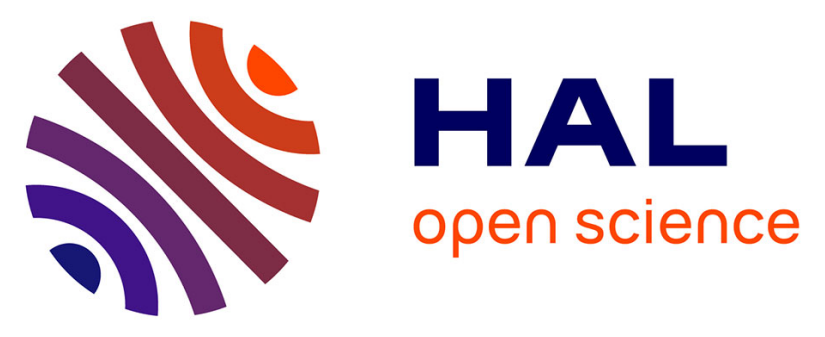

\title{
On the possibility to detect acts of cluster decay of atomic nuclei by the methods of noise diagnostic
}

\author{
Anatoli Dmitrovitch Skorbun, Oleksandr Arkadijovitch Kuchmagra, Gennadij \\ Igorevitch Odinokin, Volodymyr Trohimovitch Maslyuk, Mykola Ivanovitch \\ Romanyuk
}

\section{To cite this version:}

Anatoli Dmitrovitch Skorbun, Oleksandr Arkadijovitch Kuchmagra, Gennadij Igorevitch Odinokin, Volodymyr Trohimovitch Maslyuk, Mykola Ivanovitch Romanyuk. On the possibility to detect acts of cluster decay of atomic nuclei by the methods of noise diagnostic. 2019. hal-02390351

\section{HAL Id: hal-02390351 \\ https://hal.science/hal-02390351}

Preprint submitted on 5 Dec 2019

HAL is a multi-disciplinary open access archive for the deposit and dissemination of scientific research documents, whether they are published or not. The documents may come from teaching and research institutions in France or abroad, or from public or private research centers.
L'archive ouverte pluridisciplinaire HAL, est destinée au dépôt et à la diffusion de documents scientifiques de niveau recherche, publiés ou non, émanant des établissements d'enseignement et de recherche français ou étrangers, des laboratoires publics ou privés. 


\title{
On the possibility to detect acts of cluster decay of atomic nuclei by the methods of noise diagnostic
}

\author{
A. D. Skorbun ${ }^{1 \dagger}$, O.A. Kuchmagra ${ }^{1}$, G.I. Odinokin ${ }^{1}$, V.T. Maslyuk ${ }^{2}$, M.I. Romanyuk ${ }^{2 \dagger \dagger}$ \\ ${ }^{1}$ Institute for safety problems of nuclear power plant NAS of Ukraine. 07270, Kyiv oblast, Chornobyl, \\ Kirova str. 36-A \\ ${ }^{2}$ Institute of Electron Physics - Universitetska str., 21, 88017 Uzhhorod, Ukraine
}

PACS 21.60.Gx; 23.60.+e ; 23.90.+w; 28.41.My

Cluster decay, as a special type of radioactive decay, up to date, is widely investigated. Nevertheless, until now, this activity is restricted: from one side by the possibilities of theoretical analysis, where some success is obtained for light nuclei only; and from another side only by experiments for nuclei defragmentation in searching for fission on magic nuclei. However, standard methods of radiometry and statistical analysis have not yet been applied. Such possibility can be realized by searching for acts of cluster decay as a rare event on an array of alpha decay acts of actinides, which are recorded by industrial ionization fission chambers. The scheme of the experiment is discussed, which consists in the registration of every act of alpha decay, against the background of which it is possible to detect the presence of nuclear clusters based on isotopes of ${ }^{12,14} \mathrm{C},{ }^{20} \mathrm{O}$, and others, which can be formed in the decay of ${ }^{234,235} \mathrm{U}$. The requirements for electronics and the background conditions for such an experiment are discussed in detail.

Key words: radioactivity, cluster decay, statistical analysis, ionization fission chamber.

\section{Introduction}

The radioactivity phenomenon, which consists of the emission of elementary nuclear particles, or even individual nuclei, today is considered as a mainly intra-nuclear process. Investigation of these processes gives information about the stability of nuclear matter, the role of shell corrections, and also about the peculiarities of nucleons interaction in a nucleus. The last characteristics is especially crucial for explaining the cluster model of the nucleus [1], when, in addition to alpha particles, more complex nuclear fragments, such as ${ }^{12,14} \mathrm{C},{ }^{20} \mathrm{O}$ can be emitted $[2,3]$. There are even molecular models of a nucleus [1], which analyzed the nature and possibilities for realization ${ }^{\dagger \dagger}$ in the nucleus the cluster structures with different dimensionality.

Experimental evidence of cluster radioactivity is looking for in the analysis of nuclei fission fragments [4]. In the last time, to this task the experiments for multi-fragmentation of atomic nuclei on the high-energy particle installations $[5,6]$ are recruited. These are enough complicated and expensive experiments with am-

$\underline{\dagger}^{\dagger}$ anskorbun@gmail.com ${ }^{\dagger \dagger}$ myk.romanyuk@gmail.com 
biguous results. Nevertheless, the main problem is that they are directed to evidence of the cluster structure of fissioning nuclei, not in the cluster decay.

In our work, it is proposed to recruit to the task of cluster radioactivity investigation, in particular, the standard methods of radiometry, and statistical analysis of noise diagnostic data. In particular, one must be looking for acts of cluster decay of nuclei-actinides among acts of natural alpha decay. The main idea of our proposal is based on a hypothesis that the energy of particles as the result of cluster decay, will be proportional to their mass and, consequently, will be in several times higher, compared to the energy of alpha-particle during the standard alpha decay process. This distinction and will be a required indication of cluster decay. The other requirement is in the necessity to organize an experiment, that long set of measurements will be fulfilled, for creating vast data array on the energy of particles, which can permit to carry out a reliable statistical analysis of the results.

Technically the problem of cluster decay searching is reduced to known in nuclear physics the task to extract or fixed rare events, using standard fission ionization chambers, for instance, KNT-31-1 [7], which are intended to measure neutron fluxes and use active elements on the base of ${ }^{234,235} \mathrm{U}$. Such a device is a conventional apparatus of the system for control of the safety of nuclear power plants, and it is reliable with very wellknown characteristics.

In the article, requirements are discussed to stabilization of equipment for registrations and organizing long continuous measurements; characteristics of electronics for such type of experiments; methods of received signals treatment, necessary for ensuring the reliability of registration of cluster decay acts. It is also discussed the question of comparing cluster decay probabilities in the frames of the tunneling theory and the theory of spontaneous fission of actinides nuclei.

\section{Cluster radioactivity: from tunneling to spontaneous nucleus fission}

The term "cluster radioactivity" is applied to a phenomenon of emission, or appearance among the products of actinides nuclei transformations, high energy nucleon clusters, which are more massive, than alpha particles. Cluster radioactivity was predicted and discovered in the 80-th of the last century [1], and more than eight nuclear clusters emitted from more than 25 heavy nuclei, from ${ }^{114} \mathrm{Ba}$ to ${ }^{241} \mathrm{Am}$, have now been experimentally confirmed $[8,9]$. Cluster radioactivity is considered as a phenomenon, intermediate between alpha decay and spontaneous fission of a nucleus. Common for both of them is a barrier mechanism of realization of a final state: formation of alpha particle or ensemble of different fission fragments. In the first case it is Coulomb barrier with the height $\mathrm{B}_{\mathrm{k}} \approx 2 \mathrm{Z} / \mathrm{A}^{1 / 3}, \mathrm{MeV}$, which prevent to alpha particle tunneling; in the second one it is a potential barrier, which is determined by difference between surface and Coulomb energies of fission nucleus, and restrain transition of nucleus from spherical to ellipsoidal form (liquid drop model) in case of $\mathrm{Z}^{2} / \mathrm{A}<48$. For an explanation of cluster radioactivity, combining of adiabatic and non-adiabatic approaches are used, depending on a mass of the emitted cluster. At that, a mass $A_{f} \sim 35$ is becoming as a critical parameter [10,11]. At $A<A_{f}$, parameters of cluster radioactivity (period of cluster decay, cluster energy) are satisfactorily described in the frame of an alpha decay theory, but in another case, adiabatic mechanisms from the theory of nucleus fission 
are used. Therefore, for the problems of cluster radioactivity, it is interesting to investigate probabilities of the yield of heavy nuclei fission fragments and to establish influence factors. Such a task can be solved in the frame of after-fission approximation when particular characteristics of mass or charge distributions are determined according to the character of the thermodynamic ordering of the nucleus fission products [12]. Figure 1 demonstrates the dependences of probabilities, $\mathrm{P}$, of the yields of different fission fragments with the atomic number $\mathrm{Z}_{\mathrm{i}}$. Results obtained both in the case of different uranium isotopes at $\mathrm{t}=0.5 \mathrm{MeV}$ ("cold" fission) and for the ${ }^{234} \mathrm{U}$ isotope with different values the degree of excitation, or its nuclear temperature, t. One can see that such results are not very sensitive to the isotopic uranium composition; however, excitation parameters of the fissioning nucleus very influence them. The degree of excitation of the ${ }^{234} U$ has a particular effect on the yields of light fission fragments. For example, the transition from "cold", $\mathrm{t}=0.5 \mathrm{MeV}$ to "hot" $\mathrm{t}=2.0 \mathrm{MeV}$ fission stages increases the probability of clusters consisting, for example, of oxygen isotopes, $\mathrm{Z}=8$, in $10^{105}$, whereas for $\mathrm{Si}$ isotopes, such growth is only $10^{73}$. For $Z=6, C$ isotopes, such growth is already $10^{112}$ !

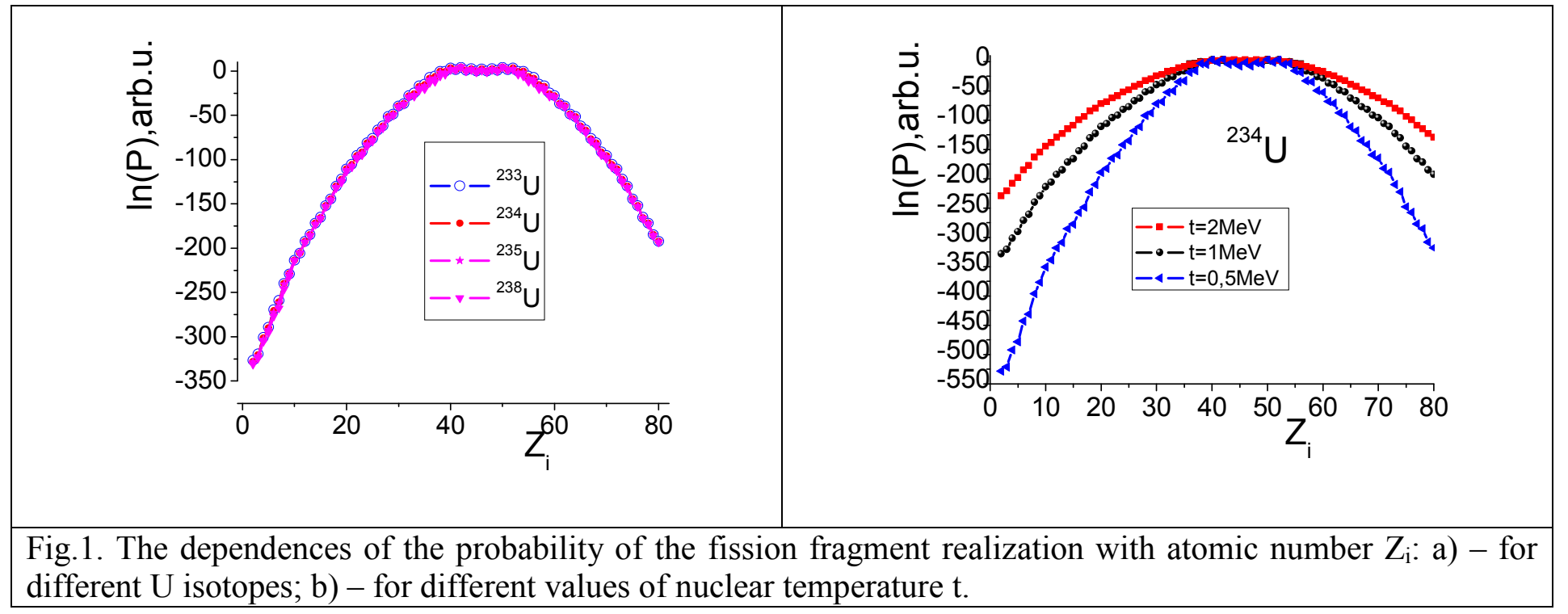

The given estimations were obtained within assumption on two-fragments fission, and not tunneling mechanism of nuclear transformations. However, in case of strong excitation of the fissioning nucleus by the high energy gamma quanta or neutron, this mechanism can give a contribution to cluster radioactivity occurrence.

\section{Characteristics of detecting system}

Schematically standard ionization fission chambers for registration of neutron flows have such a construction [7]. They are filled with gas into the working volume with two electrodes are placed there. The thin layer of ${ }^{235} \mathrm{U}$ covers one of the electrodes (cathode), and such construction is named as the radiator. Under neutron action, ${ }^{235} \mathrm{U}$ is divided, and high-energetic fragments of fission ionize a gas. If to feed high voltage to the anode, then products of ionization - electrons and ions - are collected there. They form an electrical impulse, an amplitude of which depends on the degree of ionization of the gas in working volume by fragments of ${ }^{235} \mathrm{U}$ fission. The appearance of an impulse in the electrical circuit of the detecting system corresponds to an act of neutron registration. In a given scheme of experiments about cluster radioactivity, one must pay considerable attention to the control of background conditions, or self-noise of an ionization chamber, against the background of 
which impulses from neutron registration are observed. The peculiarity of an ionization fission chamber, specifically the KNT-31-1 apparatus, which is proposed to use in the given work, is that their noises can have the two components: noises of electronics and the same, produced by spontaneous alpha-decay of uranium, coated on an electrode, so-called alpha-current or alpha-noise. This effect at neutron registration is considered as parasitic one. Furthermore, it can be easily eliminated, using the fact that the amplitude of impulse from alphaparticles is much less than from neutron impulses. Therefore, at using amplitude discriminator on the corresponding level, the system will fix only impulses, excited by neutrons.

However, for our task, an ionization chamber can be considered as an ideal source of alpha-particles, with the complete and refined system of their registration. Statistical properties of alpha-current, to use it, have been investigated in our previous works [13], where it was shown, including, that it is possible to plainly distinguish alpha-current and, correspondingly, to investigate its statistical properties.

According to this, we consider a possibility to use an alpha-decay source inside of the ionization chamber, for the task of cluster decay searching. As it was pointed out, we will proceed from the assumption that as an indication of cluster decay will be impulses, amplitudes of which will exceed amplitudes of impulses from alpha-current. In this case, such conditions correspond to the known task of registering of neutron pulses on the of $\alpha$-current's background with the difference that the amplitudes of the single pulses from the cluster decay, as expected, are comparable in order of magnitude to the amplitudes of the pulses of $\alpha$-current.

For more specific consideration, Figure 2 presents results about a neutron pulse, - a), and $\alpha$-current signals, - b), illustrating the difference in such signal detection. As one can see, the neutron pulse is observed on a nearly flat path of the panel, while the $\alpha$-current pulses must be separated from the noise background. However, it was shown in our works $[13,14]$ that electronics noise, $\alpha$-current signals, and neutron pulses can be assuredly separated.

\section{Experiments on cluster radioactivity with using noise diagnostics methods}

In experimental studies for receiving necessary input information for statistical analyzing, equipment must be used, which is capable of fixing and of storing a chronological sequence (time sets) of moments of registration of every neutron, which get into a detector, with some time resolution. It is essential to ensure automation and control of the measurement processes, raw data calculations, and processing according to developed methods and algorithms for different methods of noise diagnostics.

Such a procedure gives the possibility of carrying out independent processing of accumulated data by using different methods of statistical or correlation noise analysis. The main advantage of this approach to the measurement of noises is that analysis of data is carried over from the stage of on-line measurements to the stage of data processing that considerably simplifies and reduces the cost of the experiment, due to all measurements are carried out by the same, industrial and relatively simple equipment. Furthermore, for our task, this means the possibility to control parameters of impulses created by our equipment, in particular, their amplitude.

During alpha-current measurements, impulses are sent to amplitude analyzer, in which they are sorted into 1024 channels according to their amplitudes. It was established that in the interval from first to 50th chan- 
nels, electronics noises are registered, and signals in the area of 50-80th channels do not depend on neutron's presence or absence (Fig. 3). Thus, in the area of 50-80th channels, the alpha-current is registered (fig. 4).

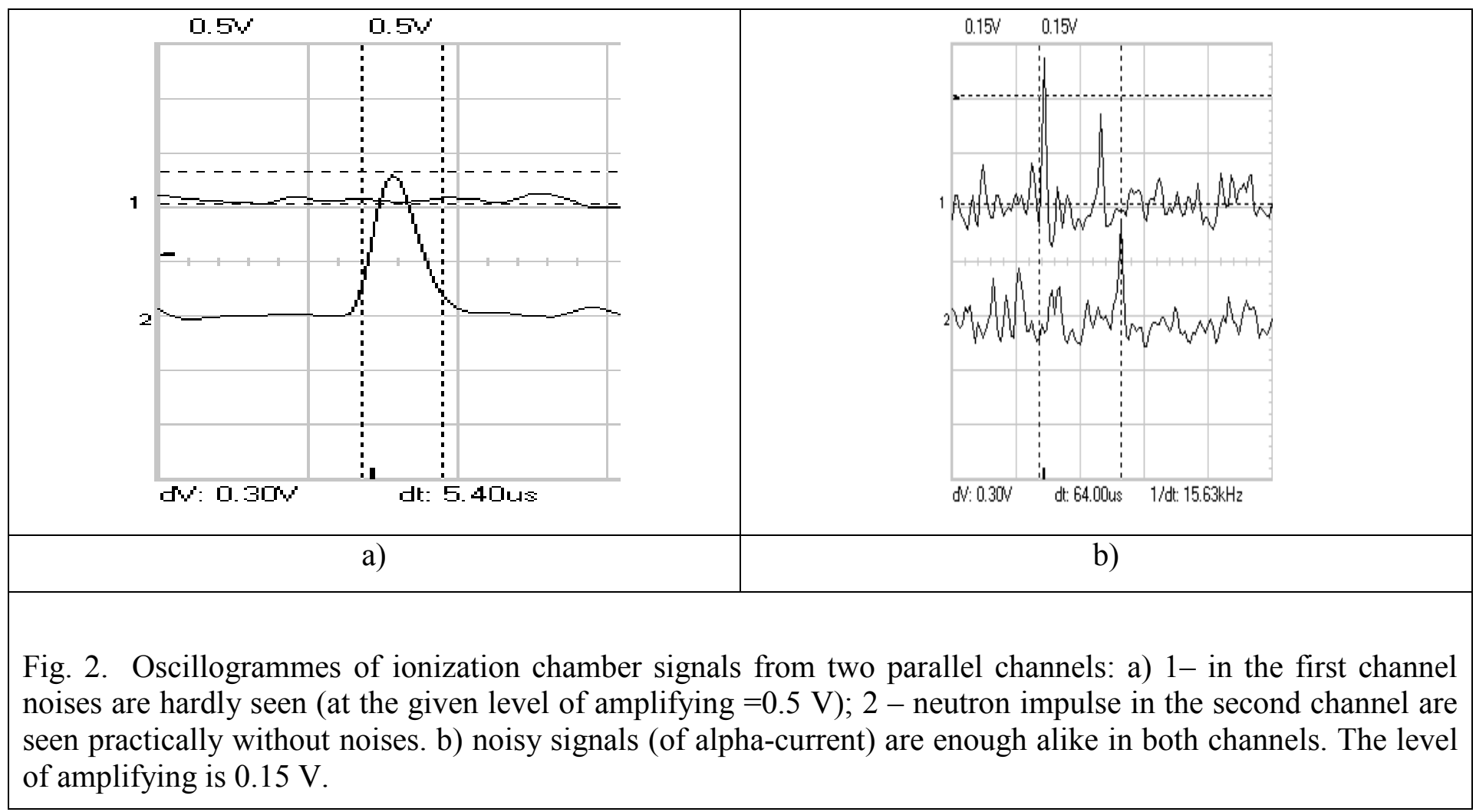

If they use two amplitude discriminators to select this area of amplitudes, it is possible to investigate statistics of alpha-current signals.

In fig. 3 the schematic image of amplitude dependences (or spectrum), which were received in the experiment with the given ionization chamber KNT-31-1, is presented. In this image it is possible to separate three areas: shaded area I, which includes 50-80th channels, where signals from detector practically do not depend on neutrons presence; also shaded area II on the right, where impulses from neutrons events are revealed; and an area from 80th to 100th channels between them with the curve 2, where alpha-current is fixed.

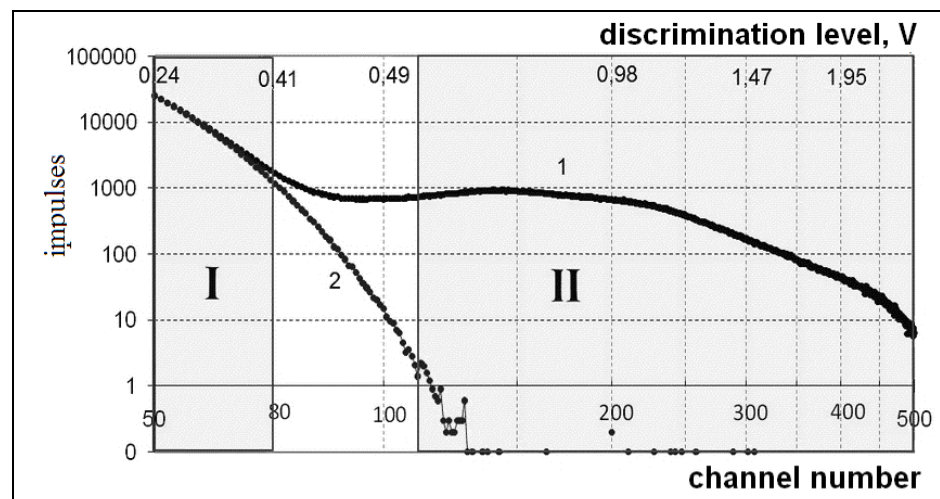

Fig. 3. Typical appearance of an amplitude spectrum for the full set of measurements with the presence of neutrons (1) and in the absence of them (2). The upper-scale demonstrates the correspondence between the level of discrimination and the number of a channel.

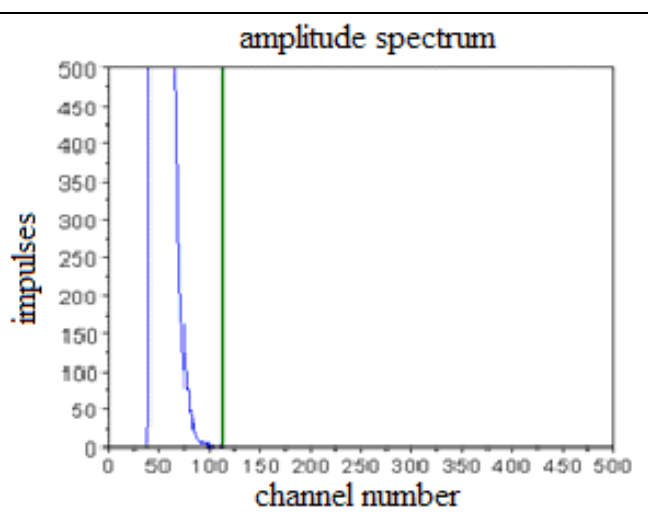

Fig. 4. The amplitude spectrum of impulses, which were registered in 50-80-th channels: the discriminator "cuts" impulses with amplitudes, less than the 50-th channel, and greater than 100-th channel (shown by the vertical line). 
As it was pointed earlier, due to some time is necessary to collect electrons and ions from ionization inside a fission chamber ("dead time"), the system can also register impulses of double or more amplitude, if ionization will be due to particles, which ionize the gas practically simultaneously, that is during a dead time. Then signals from two or more alpha-particles will be received as a single one.

The probability of such processes can be estimated if to consider them as Poisson ones. In our case, it is suggested that the time of ionization charges collection in chamber KNT-31- 1 is equal to $\tau=0.4 \cdot 10^{-6} \mathrm{~s}$. Estimating the probability of occurrence of overlaps allows one to determine the number of expected events. It is possible to estimate the probability of events overlaps for alpha-particles during the time of measurement $\mathrm{t}$ according to the formula [15]:

$$
P_{n}(n, \tau)=\frac{\left(n_{0} \cdot \tau\right)^{n}}{n !} \cdot e^{-n_{0} \cdot \tau}
$$

where

- $\mathrm{n}$ is the number of alpha-particles, which create overlap;

- $\mathrm{n}_{0}$ is the number of alpha-particles, which is emitted by radiator in one second inside of fission chamber volume;

- $\tau$ is the time, during which the overlap is possible.

Then the number of overlapping for time unit is calculated according to formula $N=n_{0} \cdot p_{n}(n \cdot \tau)$.

Table 1. Results of the standard calculations of the probability of simultaneous registration of alpha-particles.

\begin{tabular}{|c|c|c|c|c|}
\hline $\mathrm{n}$ & $\frac{\left(n_{0} \cdot \tau\right)^{n}}{n !}$ & $e^{-n_{0} \cdot \tau}$ & $p_{n}$ & $N$ \\
\hline 1 & 0.25 & 0.778 & 0.1945 & 121562 \\
\hline 2 & 0.03125 & 0.778 & 0.0243 & 15187 \\
\hline 3 & $2.6 \cdot 10^{-3}$ & 0.778 & $2.0 \cdot 10^{-3}$ & 1250 \\
\hline 4 & $1.63 \cdot 10^{-4}$ & 0.778 & $1.27 \cdot 10^{-4}$ & 79 \\
\hline 5 & $8.14 \cdot 10^{-6}$ & 0.778 & $6.33 \cdot 10^{-6}$ & 4 \\
\hline
\end{tabular}

Thus calculations show that in ionization chamber KNT-31-1, the impulses from overlapping up to five alpha-particles can arise. It is considered that such summing impulses, in reality, are fixed by existing equipment. Their number is decreased with the increase of the amplitude of a summing impulse, hereupon an amplitude spectrum in fig.3 is not the straight line, but a diffuse area from 50-th to 100-th channels. According to reference data for ${ }^{234} \mathrm{U}$, which also is present on the cathode of KNT-31-1, it is an intensive source of alphaparticles, which have energy $4722.4 \mathrm{keV}$ (in $28,42 \%$ cases) and $4774.6 \mathrm{keV}$ (in $71.38 \%$ cases). In the case of five alpha-particle overlapping and full ionization charge accumulation inside an ionization chamber, the impulse with energy about $30 \mathrm{MeV}$ is registered. This energy is comparable with the energy of a light nucleus at cluster radioactivity [2]. The amplitude of such an impulse is belonged to the range of values for fission fragment registration in the case of neutron detecting and can be fixed in an amplitudes spectrum.

One can try to avoid this problem, taking into account the peculiarity of the fission chamber, that a radiator of KNT-31-1 chamber, in reality, is a mixture of ${ }^{235} U+{ }^{234} \mathrm{U}$ isotopes. The activity of ${ }^{234} \mathrm{U}$, despite its 
small concentration, is higher by several orders of magnitude than ${ }^{235} \mathrm{U}$. That is noise current or alpha-current of such chambers is mainly the result of ${ }^{234} \mathrm{U}$ decay.

Therefore, for the experiments on cluster radioactivity searching, to reduce the number of background impulses, one should use the fission chamber [16], in which the radiator must be of exceptionally high enrichment $\left(99.9 \%{ }^{235} \mathrm{U}\right.$, less than $\left.0.03 \%{ }^{234} \mathrm{U}\right)$. Such a sort of fission chamber can be more expensive, but the intensity of alpha-current in them is much lesser. In this case, the probability the overlapping of impulses of $\alpha$ particles under the experimental conditions decreases and increases the expectation for the possibility of registration of cluster particles.

Others possibilities for the success of such an experiment are as follows:

1. Equipment modernization, which consists of creating high-quality electronics for registration impulses from the ionization fission chamber, which reduces an electronics component of dead time to the level of physical restrictions for the processes of ionization and charge collection, which we estimate as $\sim 400 \mathrm{~ns}$.

2. The necessity of theoretical modeling of multi-particle registration of alpha-current impulses, which should base on experimental data.

3. New theoretical investigations are necessary for the systematization of fission fragments of actinides. It is mainly concern the part of the selection and estimation of the probability of yields of the light magic nuclei. It is necessary also to take into account the different versions of cluster decay mechanism.

4. Because the matter is of registration of single events, in the modernized experimental setup, the measures must be taken to reducing and stabilization of background conditions in detecting system from neutron impulses, the equipment stabilization, and to provide long-time measurements.

\section{Conclusions}

Thus, registration of acts of atomic nuclei cluster decay by the noise diagnostics methods must take into account the fact that such events will be observed as an integral part of alpha decay, including the presence of overlapping processes during measurements. The effect of overlapping impulses from practically simultaneously registered alpha particles seems to be big enough, and it will be not simply to register the cluster decay impulses against this background. As an option, it is proposed to use ionization chambers, which have as a radiator's material the isotope ${ }^{235} \mathrm{U}$, enriched comparatively to ${ }^{234} \mathrm{U}$. An essential item is decreasing of dead time of registering equipment employing by using high-quality electronics. It is necessary also to carry out numerical estimations of amplitude spectral lines broadening, taking into account alpha particle energy at multi-particles impulses overlapping. The proposed approach can also bring success for the registration of more massive nuclei clusters, such as ${ }^{34} \mathrm{Si}$, in the case of their emission from uranium.

A special task is the stabilization of power supply units and ensuring low background conditions of detecting system from neutrons in case of long-time measurements. 


\section{References}

1. Sandulescu, A., Poenaru, D. N. and Greiner W. New type of decay of heavy nuclei intermediate between fission and alpha-decay". Sov. J. Part. Nucl. 11, pp. 528-541 (1980).

2. Bonetti, R. and Guglielmetti, A., Cluster radioactivity: an overview after twenty years, Rom. Rep. Phys. 59 (2007) 301-310.

3. Seif W.M., Amer L.H. Systematic investigation of cluster radioactivity for uranium isotopes. Nuclear Physics A Vol. 969.- p. 254-268

4. Kuklin S. N. , Adamian G. G. , Antonenko N. V. Description of alpha decay and cluster radioactivity in the dinuclear system model. Physics of Elementary Particles and Atomic Nuclei. - 2016.- V. 47, Issue 2, p. 206-235.

5. Tohru Motobayashi, Hiroyoshi Sakurai. Research with fast radioactive isotope beams at RIKEN. Progress of Theoretical and Experimental Physics, Volume 2012, Issue 1, 2012, 03C001, https://doi.org/10.1093/ptep/pts059.

6. Bezbakh A. N. et al. Quasiparticle Structure of Superheavy Nuclei along the $\alpha$-Decay Chain of 288/115. Phys. Rev. C. 2015. V. 92. P. 014329.

7. Mitelman M. G., Dubovskii V. G., Liubchenko V. F., Rosenblum N. D. Detectors for intrareactor measurements of energy release. Moskva: Energoatomizdat, 1977, 152 p. (in russian)

8. K.N. Mukhin, O.O. Patarakin. Exotic processes in nuclear physics. Phys. Usp. $43799-839$ (2000) (in russian).

9. Poenaru D., Greiner W., Cluster Radioactivity, Ch. 1 of Clusters in Nuclei I. Lecture Notes in Physics818 (Springer, Berlin, 2011), Ed. C. Beck, pp. 1-56, ISBN 978-3-642-13898-0.

10. Kuklin S.N., Adamian G.G., Antonenko N.V. Description of Alpha Decay and Cluster Radioactivity in the Dinuclear System Model. Physics of Elementary Particles and Atomic Nuclei. -2016.- V. 47. \# 2, pp. 390-441(in russian)

11. Ogloblin A. A. et al. Observation of cluster decay of 242Cm. Phys. Rev. C.- 2000.- V.61, 034301.

12. Maslyuk V.T., et al. Calculation of the fission-fragment yields of the pre-actinide by the example of the natPb isotopes. Nuclear Physics A.-2016 955 (2016) 79.

13. O. A. Kuchmagra, G. I. Odinokin, A. D. Skorbun, S. M. Stadnyk, A. S. Sadovnikov. Using of alphaactivity of fission chamber radiator for control of workability of neutron measurement channel. Problemy bezpeky atomic station and Chornobyl.- 2006.- V.27, pp. 97-101 (in ukrainian)

14. O. A. Kuchmagra, I.O. Maznyi, G. I. Odinokin, A. S. Sadovnikov, A. D. Skorbun, S. M. Stadnyk. Fission Ionization Chamber as Reference Source in Neutron Flux Analysis. Nuclear and radiation safety. 2017. - V.4.- \#76. P. 102-106 (in ukrainian).

15. Kalashnikova, M. S. Kozodayev. Detectors of elementary particles. Moskva. Nauka. 1996. 407 p. (in russian).

16. B. Dmitriev, E.K. Malishev. Neutron ionization chambers for reactor technics. Atomizdat. Moskva, 1975. 94 p. (in russian). 